CLINICAL STUDY

\title{
Acylated and nonacylated ghrelin levels and their associations with insulin resistance in obese and normal weight children with metabolic syndrome
}

Lucia Pacifico, Eleonora Poggiogalle, Francesco Costantino, Caterina Anania, Flavia Ferraro, Francesco Chiarelli ${ }^{1}$ and Claudio Chiesa ${ }^{2}$

Department of Pediatrics, Sapienza University of Rome, Viale Regina Elena, 324, 00161 Rome, Italy, ${ }^{1}$ Department of Pediatrics, University of Chieti, 66100 Chieti, Italy and ${ }^{2}$ National Research Council, 00161 Rome, Italy

(Correspondence should be addressed to L Pacifico; Email: lucia.pacifico@uniroma1.it)

\begin{abstract}
Background: Ghrelin, a peptide mainly derived from the stomach, plays a pivotal role in the regulation of food intake, energy metabolism, and storage, as well as in insulin sensitivity. Ghrelin circulates in acylated (A-Ghr) and nonacylated (NA-Ghr) forms, and their potential differential associations with insulin resistance (IR) in childhood obesity remain undefined.

Objective: We investigated the associations of ghrelin forms with IR in normal weight and obese children and the impact of metabolic syndrome (MS) on their plasma values.

Design: A total of 210 children in four subgroups of normal weight/obese children with and without components of MS were studied. Fasting blood glucose, insulin, lipid profile, and acylated and total ghrelin were examined. IR was determined by a homeostasis model assessment (HOMA) of IR.

Results: In the entire population, plasma insulin and HOMA-IR were associated negatively with T-Ghr and NA-Ghr, but positively with the ratio of A/NA-Ghr after adjustment for age, gender, and Tanner stage. Obese metabolically abnormal children had lower T-Ghr and NA-Ghr, but comparable A-Ghr and a higher A/NA-Ghr ratio than obese metabolically normal subjects. Compared with lean healthy children, lean metabolically abnormal subjects had higher A-Ghr and the A/NA-Ghr ratio, but comparable T-Ghr and NA-Ghr. A multiple regression analysis showed that A-Ghr and the A/NA-Ghr ratios were positively associated with HOMA-IR, independent of age, gender, Tanner stage, and body mass index (or waist circumference) and other components of MS.

Conclusions: A-Ghr excess may negatively modulate insulin action in obese and nonobese children, and may contribute to the association of IR and MS.
\end{abstract}

European Journal of Endocrinology $161861-870$

\section{Introduction}

Ghrelin, a 28-amino acid peptide predominantly produced by the stomach, is expressed in many other central and peripheral tissues including hypothalamus, liver, kidney, pituitary gland, endocrine pancreas, and adipose tissue $(1,2)$. In addition to potent GH-releasing activity, ghrelin is involved in the control of food intake and energy metabolism at central levels as well as at peripheral levels influencing the endocrine pancreatic function and glucose and lipid metabolism $(3,4)$. In humans, circulating levels of ghrelin are increased in anorexia and cachexia, but are reduced in obesity, insulin resistance (IR) and type 2 diabetes. $(1,4-6)$. Several in vitro and in vivo studies have shown that ghrelin may regulate insulin and glucose metabolism (7-11). Ghrelin was shown to inhibit insulin secretion from pancreatic islets in rodents (9). Intravenous administration of ghrelin to healthy young men impaired insulin sensitivity (10). In obese women with polycystic ovary syndrome, ghrelin induced a significant decrease in insulin concentrations; an increase (though not significant) in glucose levels was also observed (11). Earlier studies on ghrelin and energy balance and metabolism were performed by total ghrelin assays. However, two forms of ghrelin have been described recently: acylated ghrelin (A-Ghr) and nonacylated ghrelin (NA-Ghr). NA-Ghr is the major circulating form and constitutes $80-90 \%$ of circulating ghrelin. Although it was originally thought that NA-Ghr lacked endocrine and biological actions, more recent findings suggest that both A-Ghr and NA-Ghr may mediate peripheral biological actions; indeed, there is a suggestion that both may act antagonistically $(1,3,12-16)$. Some studies have provided evidence that A-Ghr and NA-Ghr have different effects on insulin metabolism. Gauna et al. reported that glucose output by primary hepatocytes is stimulated by A-Ghr and is inhibited by 
NA-Ghr (13). Broglio et al. demonstrated that NA-Ghr counteracted the stimulatory effect of A-Ghr on glucose release, and that it was able to antagonize the influence of A-Ghr on insulin secretion and glucose metabolism (14). Under pharmacological concentrations, A-Ghr induced IR, whereas a combination of NA-Ghr and A-Ghr improved insulin sensitivity $(15,16)$. These results suggest that A-Ghr may act as a diabetogenic factor and may therefore potentially modulate a deterioration of insulin sensitivity.

The relation of A-Ghr to NA-Ghr may also have a clinical significance in metabolic disorders as it has been demonstrated recently in adults. Obesity altered circulating ghrelin profile, and relative A-Ghr excess was suggested to contribute to obesity-associated IR in metabolic syndrome (MS) (17). Yet, both increased A-Ghr physiological concentrations, and elevated A/NA-Ghr ratios were associated with IR in obese and overweight postmenopausal women (18). Taken together, these findings provide some evidence of differential associations of ghrelin forms with insulin action in obese adults. However, relatively little information exists in obese children $(19,20)$. There is ample evidence suggesting that the complications of obesity seen with adults begin in early childhood $(21,22)$. IR, hypertension, glucose intolerance, and type 2 diabetes mellitus have all been associated with childhood obesity $(23,24)$. The concomitant occurrence of these abnormalities, referred to as MS, accrues a significant risk for the development of cardiovascular disease and diabetes during early adulthood, if not during childhood. Obesity in children is in rapid expansion across the world, and studies in this age group are important.

Therefore, the aims of this study were to explore in children i) the associations of ghrelin secretion profiles, including T-Ghr, A-Ghr, NA-Ghr, and the A/NA-Ghr ratio, with IR independently of obesity, as well as of other components of MS; and ii) the impact of MS and its single components on ghrelin forms.

\section{Methods}

\section{Subjects}

Over a 15-month period, children and adolescents with primary obesity (body mass index (BMI) equal to or higher than the age- and sex-specific 97th percentile (25)) were consecutively enrolled at the Department of Pediatrics, Sapienza University of Rome, if they met the following criteria: no renal disease; no type 1 or 2 diabetes; no condition known to influence body composition, insulin action, or insulin secretion (e.g. glucocorticoid therapy, hypothyroidism, and Cushing's disease); and no history of alcohol consumption and smoking (where appropriate). At enrollment, their visit included physical examination (i.e. the measurements of weight, standing height, waist circumference (WC), and BMI, and the determination of the stage of puberty according to the criteria of Tanner $(26,27))$, measurements of body composition (including the determination of lean body mass and total fat mass), and laboratory tests. WC was obtained at the midpoint between the lowest rib and the iliac crest. The measurement was made at the end of a normal expiration while the subjects were in a standing position. The degree of obesity was quantified using Cole's least mean square method, which normalizes the skewed distribution of BMI and expresses BMI as a SDS. This measure gives age- and sex-specific estimates of the distribution median, the variation coefficient, and the degree of skew by a maximum-likelihood fitting technique (28). Systolic blood pressure (BP) and diastolic BP were measured twice at the right arm after a 10-min rest in the supine position using an automated oscillatory system (Dinamap Vital Signs Monitor, Model 1846 SX; Criticon Incorporated, Tampa, FL, USA). Body composition was determined using a total body scanner (Hologic QDR-4500W, Waltham, MA, USA, which uses fan-beam scanning) in array mode. This equipment uses a switched pulse stable dual energy X-ray operating at 100 and $140 \mathrm{kV}$. The data were analyzed using the software version 11.2.

Over the same study period, normal weight children, defined as those with BMI appropriate for gender and age (BMI $<85$ th), were recruited to the study if they had no history of chronic disease; no history of use of medications that would affect insulin action or insulin secretion; no history of alcohol consumption and smoking (where appropriate); and their parents consented to participate in the study. At enrollment, their visit included physical examination (i.e. the measurements of weight, standing height, $\mathrm{WC}$, and BMI, and the determination of the stage of puberty), measurements of body composition, and laboratory tests.

Both obese and normal weight children denied any significant change in weight during the last 3 months prior to the enrollment in the study.

The study was approved by the hospital ethics committee, and informed consent was obtained from the subjects' parents prior to the assessment.

\section{Laboratory data}

Blood samples were taken from each subject after an overnight fast for the measurement of circulating concentrations of glucose, insulin, triglycerides, total cholesterol and high-density lipoprotein (HDL) cholesterol, T-Ghr, and A-Ghr. In order to prevent the degradation and loss of the octanoyl group on the serine 3 position of ghrelin after centrifugation, A-Ghr samples were processed with $50 \mu \mathrm{l}$ of $1 \mathrm{M} \mathrm{HCl}$ and with the addition of $10 \mu \mathrm{l}$ phenylmethylsulfonyl fluoride per $1 \mathrm{ml}$ plasma. Plasma immunoreactive T-Ghr and A-Ghr 
values were measured in duplicate with commercial RIA using ${ }^{125}$ I-labeled bioactive human A-Ghr as a tracer and a rabbit polyclonal antibody raised against full-length T-Ghr and against the serine 3 position of the octanoylated portion of A-Ghr respectively (Linco Research, Inc., St Charles, MO, USA). According to the supplier's specifications, inter- and intra-assay coefficient of variations (CV) were 14.7-17.8 and 3.3-10.0\% respectively for T-Ghr, and were 9.6-16.2 and 6.5-9.5\% respectively for A-Ghr. NA-Ghr values were calculated as T-Ghr-A-Ghr $(17,18)$. Insulin concentrations were determined using RIA with polyclonal antibodies (CIS Bio International, Schering SA, Gif-SurYvette Cedex, France; detection limit, $2.0 \mathrm{mU} / \mathrm{l}$; interand intra-assay CV, 6.4-8.8 and 4.2-8.2\% respectively). We measured the remaining analytes on a COBAS INTEGRA 800 analyzer (Roche Diagnostics). Total cholesterol, HDL cholesterol, and triglyceride concentrations were assessed with the cassettes COBAS INTEGRA total cholesterol version 2; HDL cholesterol was assessed with version 3; triglyceride was assessed according to International Federation of Clinical Chemistry (IFCC) respectively by enzymatic colorimetric methods; and glucose concentration was assessed with the cassette version 3 by a hexokinase method (Roche Diagnostics).

\section{Definitions}

Because there is still no universally accepted definition of MS in children and adolescents, the criteria used in pediatric studies have been variably adapted from adult standards with the use of gender- and age-dependent normal values (29-34). For the American Heart Association (AHA) definition, MS is diagnosed in the presence of any three of the following five constituent risks: obesity as determined by WC; hypertension; low HDL values; elevated triglyceride values; glucose impairment (34). Our definition was based on the AHA definition, but it used pediatric reference standards for $\mathrm{BP}, \mathrm{WC}$, triglycerides, and HDL cholesterol. The glucose cutoff was identical to that used in the AHA definition. In our study, obesity was defined as a BMI $\geq 97$ th percentile adjusted for age and sex (25) and/or a WC $\geq 90$ th percentile for age and sex (35); hypertriglyceridemia as triglycerides $\geq 95$ th percentile for age and sex (36); low HDL cholesterol as concentrations $\leq 5$ th percentile for age and sex (36); elevated BP as systolic or diastolic BP $\geq 95$ th percentile for age and sex (37); and impaired fasting glucose as glucose $\geq 5.6 \mathrm{mmol} / \mathrm{l}(26)$. IR was determined by a homeostasis model assessment (HOMA) of IR. Scores were calculated as the product of the fasting serum insulin concentration $(\mathrm{mU} / \mathrm{l})$ and the fasting serum glucose concentration $(\mathrm{mmol} / \mathrm{l})$ divided by 22.5 . HOMA-IR changes during childhood depended on the age, gender, and pubertal stage (38). We have considered HOMA-IR values $\geq 95$ th percentile as an indicator of
IR. We therefore defined MS in the presence of any three of the following five constituent risks: obesity; hypertension; low HDL cholesterol; elevated triglycerides; impaired fasting glucose and/or IR.

\section{Statistical analysis}

Statistical analyses were performed by SPSS (version 13.0; SPSS Inc, Chicago, IL, USA). Data are expressed either as frequencies or as means with $95 \%$ confidence intervals (CIs). Distributions of continuous variables were examined for skewness and kurtosis, and were logarithmically transformed when appropriate. Geometric means are reported for ghrelin forms, insulin, and HOMA-IR values. Differences between groups were tested for significance using ANOVA for quantitative variables with the Bonferroni correction for multiple comparisons, and using $\chi^{2}$ test for qualitative variables. Pearson's correlation and linear regression coefficients were used to examine the relationship between variables. First, multiple linear regression analyses were performed to assess the associations of HOMA-IR values with ghrelin forms (considered one at a time) after adjustment for age, gender, Tanner stage, and BMI (or WC) (model 1); and second, the model was adjusted for age, gender, Tanner stage, BMI (or WC), and other components of MS including triglycerides, HDL cholesterol, and systolic BP. Log HOMA-IR, log T-Ghr, log A-Ghr, log NA-Ghr, and the log A/NA-Ghr ratios were used for correlations, and log HOMA-IR was used as the dependent variable in multiple regression analyses. $P$ values $<0.05$ were considered statistically significant.

\section{Results}

\section{Clinical and biochemical characteristics of the study population}

A total of 210 children and adolescents were included in the study. Participants were 34 obese children with normal blood glucose, insulin concentrations, lipid profile, and BP considered as the phenotypically obese metabolically normal (POMN) group; 79 with one component or more components of MS, the phenotypically obese metabolically abnormal (POMA) group; 55 normal weight children with normal blood glucose, insulin values, lipid profile, and BP, the normal weight metabolically normal (NWMN) group; and 42 children with normal weight and with one component or more components of MS, the normal weight metabolically abnormal (NWMA) group.

Baseline anthropometric and metabolic characteristics of the four groups of participants are presented in Table 1. Children in the POMA group were older than those in the POMN and NWMA groups, and had higher BMI, total fat, and lean mass than the other groups. BMI-SDS as well as systolic BP and diastolic BP were higher in obese children than in normal weight 
Table 1 Anthropometric and metabolic characteristics of obese and normal weight children

\begin{tabular}{|c|c|c|c|c|}
\hline & POMA $(n=79)$ & POMN $(n=34)$ & NWMA $(n=42)$ & NWMN $(n=55)$ \\
\hline Age (year) & $10.0(9.4-10.7)^{\star, ~ a ~}$ & $8.7(7.7-9.6)$ & $8.4(7.2-9.6)$ & $9.3(8.3-10.2)$ \\
\hline Male, $n(\%)$ & $44(56)$ & $22(65)$ & $23(55)$ & $24(44)$ \\
\hline \multicolumn{5}{|l|}{ Tanner stage, $n(\%)$} \\
\hline I & $27(34)$ & $19(56)$ & $30(72)$ & $27(49)$ \\
\hline II-II & 25 (32) & $13(38)$ & $9(21)^{\prime}$ & $21(38)$ \\
\hline IV-V & $27(34)$ & $2(6)$ & $3(7)$ & $7(13)$ \\
\hline BMI $\left(\mathrm{kg} / \mathrm{m}^{2}\right)$ & $26.0(25.3-26.8)^{\ddagger, \uparrow, b}$ & $23.7(22.6-24.8)^{ \pm, ~ ๆ ~}$ & $16.0(15.5-16.6)$ & $16.6(16.0-17.1)$ \\
\hline BMI-SDS & $2.16(2.0 \text { to } 2.3)^{ \pm, ~}$ & $2.11(2.0-2.2)^{\ddagger}, \Phi$ & $-0.09(-0.32-0.13)$ & $-0.12(-0.33-0.08)$ \\
\hline Waist circumference $(\mathrm{cm})$ & $81.1(78.2-83.7)^{\ddagger, ~ ๆ ~}$ & $80.9(78.2-83.7)$ & $67.1(63.9-70.4)^{\S}$ & $61.8(58.0-65.6)$ \\
\hline Systolic BP (mmHg) & $115(113-116)^{ \pm, \cdot \pi}$ & $115(113-117)^{\ddagger},{ }^{\prime \prime}$ & $107(105-108)$ & $105(104-107)$ \\
\hline Diastolic BP (mmHg) & $75(72-76)^{\ddagger}, \uparrow^{\prime}$ & $75(72-77)^{\ddagger}, q^{\prime}$ & $66(65-68)$ & $65(64-67)$ \\
\hline Total fat mass $(\mathrm{kg})$ & $20.6(18.8-22.4)^{\ddagger, \tau, b}$ & $15.4(13.2-17.6)^{\ddagger}$. ๆ & $4.6(3.8-5.8)$ & $5.7(4.7-6.7)$ \\
\hline Total lean mass (kg) & $30.3(28.0-32.6)^{\ddagger,}$ 甲, b & $23.5(20.6-26.4)^{\dagger}, \S$ & $16.2(13.9-18.5)$ & $19.0(16.8-21.3)$ \\
\hline Total cholesterol (mg/dl) & $177(165-190)^{\star,} \|$, a & $149(138-160)^{\ddagger}$ & $194(178-210)^{\top}$ & $151(145-157)$ \\
\hline HDL cholesterol (mg/dl) & $41(39-43)^{\star \Uparrow}$ & $45(43-48)^{\S}$ & $46(42-50)^{\S}$ & $53(49-57)$ \\
\hline Triglycerides $(\mathrm{mg} / \mathrm{dl})$ & $135(110-161)^{\star, ~ ๆ, ~ c ~}$ & $72(62-82)$ & $95(77-114)^{\S}$ & $60(53-65)$ \\
\hline Fasting glucose $(\mathrm{mmol} / \mathrm{l})$ & $4.82(4.72-4.91)^{a}$ & $4.59(4.45-4.73)$ & $4.70(4.53-4.84)$ & $4.72(4.62-4.82)$ \\
\hline Fasting insulin $(\mathrm{mU} / \mathrm{l})$ & $19.0(17.0-21.3)^{\ddagger, \uparrow, c}$ & $10.4(9.0-12.2)^{?}$ & $9.3(7.4-11.7)^{\| \prime}$ & $6.2(5.4-7.1)$ \\
\hline HOMA-IR values & $4.0\left(3.6-4.6^{\neq,}, \tau^{\prime}, \mathrm{c}\right.$ & $2.1(1.8-2.4)^{\| \prime}$ & $1.9(1.5-2.4)^{\|\|^{\prime}}$ & $1.3(1.1-1.5)$ \\
\hline Total ghrelin (ng/l) & $208(183-241)^{\ddagger}$, ๆ, a & $302(243-376)^{\star \S}$ & $403(330-487)$ & $428(354-513)$ \\
\hline Acylated ghrelin (ng/l) & $78(70-89)^{\ddagger}$ & $81(69-93)^{\dagger}$ & $110(92-136)^{11}$ & $84(74-96)$ \\
\hline Nonacylated ghrelin (ng/l) & $97(76-125)^{\dagger, ~ \uparrow, ~ a ~}$ & $171(109-269) \S$ & $238(166-340)$ & $318(251-403)$ \\
\hline $\begin{array}{l}\text { Acylated to nonacylated } \\
\text { ghrelin ratio }(\%)\end{array}$ & $81(60-110)^{\star, ~ \uparrow, ~ a ~}$ & $47(29-76)^{\S}$ & $47(31-71)^{\S}$ & $26(21-34)$ \\
\hline
\end{tabular}

ANOVA with the Bonferroni correction for multiple comparisons: ${ }^{*} P<0.05,{ }^{\dagger} P<0.001,{ }^{\ddagger} P<0.0001$ versus NWMA; ${ }^{\S} P<0.05, "{ }^{1} P<0.001,{ }^{~} P<0.0001$ versus NWMN; ${ }^{\mathrm{a}} P<0.05,{ }^{\mathrm{b}} P<0.001,{ }^{\mathrm{c}} P<0.0001$ versus POMN.

Results are expressed as $n(\%)$, mean $(95 \% \mathrm{Cl})$, or geometric mean $(95 \% \mathrm{Cl})$ for log-transformed variables. POMA, phenotypically obese metabolic abnormal; POMN, phenotypically obese metabolic normal; NWMA, normal weight metabolically abnormal; NWMN, normal weight metabolically normal.

subjects, but were similar within the same weight category. WC was higher in obese children than in normal weight subjects, but was similar in the POMA and POMN groups. In contrast, WC was higher in the NWMA group than in the NWMN group. Mean values of triglycerides, insulin, and HOMA-IR were significantly higher in the POMA group than in the other three groups. Yet, within the normal weight category, the NWMA children had higher triglycerides, insulin, and HOMA-IR values than the NWMN group. The NWMA group had higher total cholesterol followed respectively by the POMA, POMN, and NWMN groups, while the POMA patients had lower HDL cholesterol followed respectively by the POMN, NWMA, and NWMN children. T-Ghr and NA-Ghr were significantly lower in the POMA children than in the other groups, while the A/NA-Ghr ratio was significantly higher. In contrast, A-Ghr levels were significantly higher in the NWMA children than in the other groups.

\section{Ghrelin profiles in relation to clinical, anthropometric, and biochemical parameters}

Within the entire study population, after adjustment for age, gender, and Tanner stage, insulin levels and HOMA-IR values were negatively related with T-Ghr

Table 2 Partial correlation coefficients between total, acylated, nonacylated, and acylated to nonacylated ghrelin ratio profiles and anthropometric and metabolic variables within the entire study population adjusted for age, gender, and Tanner stage.

\begin{tabular}{|c|c|c|c|c|}
\hline & Total ghrelin & Acylated ghrelin & Nonacylated ghrelin & $\begin{array}{l}\text { Acylated to nonacylated } \\
\text { ghrelin ratio }\end{array}$ \\
\hline BMI $\left(\mathrm{kg} / \mathrm{m}^{2}\right)$ & $-0.374^{\ddagger}$ & -0.126 & $-0.349^{\ddagger}$ & $0.262^{\ddagger}$ \\
\hline Waist circumference $(\mathrm{cm})$ & $-0.234^{\dagger}$ & 0.013 & $-0.254^{\dagger}$ & $0.233^{\dagger}$ \\
\hline Total fat mass $(\mathrm{kg})$ & $-0.341^{\ddagger}$ & -0.121 & $-0.300^{\ddagger}$ & $0.221^{\dagger}$ \\
\hline Total lean mass (kg) & $-0.374^{\ddagger}$ & $-0.217^{\dagger}$ & $-0.329^{\ddagger}$ & $0.206^{\dagger}$ \\
\hline Systolic BP (mmHg) & $-0.273^{\ddagger}$ & -0.022 & $-0.298^{\ddagger}$ & $0.258^{\ddagger}$ \\
\hline Triglycerides (mg/dl) & $-0.256^{\ddagger}$ & -0.095 & $-0.202^{\ddagger}$ & $0.142^{*}$ \\
\hline Total cholesterol (mg/dl) & $-0.153^{\star}$ & 0.083 & $-0.142^{\star}$ & $0.161^{\star}$ \\
\hline HDL cholesterol (mg/dl) & $0.229^{\dagger}$ & 0.044 & $0.204^{\dagger}$ & $-0.166^{\star}$ \\
\hline Fasting glucose (mmol/l) & -0.099 & -0.016 & -0.113 & 0.095 \\
\hline Fasting insulin $(\mathrm{mU} / \mathrm{l})$ & $-0.292^{\ddagger}$ & 0.016 & $-0.296^{\ddagger}$ & $0.273^{\ddagger}$ \\
\hline HOMA-IR values & $-0.303^{\ddagger}$ & 0.013 & $-0.309^{\ddagger}$ & $0.284^{\ddagger}$ \\
\hline
\end{tabular}

${ }^{\star} P<0.05 ;{ }^{\dagger} P<0.001 ;{ }^{\ddagger} P<0.0001$. 
and NA-Ghr, whereas they were positively associated with the A/NA-Ghr ratio (Table 2). BMI, WC, lean and fat mass, and systolic BP and diastolic BP, as well as total cholesterol and triglycerides were correlated negatively with T-Ghr and NA-Ghr, but positively with the A/NA-Ghr ratio also after adjusting for age, gender, and Tanner stage (Table 2). In contrast, HDL cholesterol was correlated positively with T-Ghr and NA-Ghr but negatively with the A/NA-Ghr ratio. A-Ghr was not significantly correlated with metabolic parameters, including insulin and HOMA-IR. Of the anthropometric characteristics, lean mass was significantly and negatively associated with A-Ghr.

When the associations were restricted to the normal weight groups, insulin values and HOMA-IR remained significantly negatively associated with T-Ghr $(r=-0.256, \quad P<0.05$, and $r=-0.259, \quad P<0.05$ respectively) as well as with NA-Ghr $(r=-0.344$, $P<0.01$, and $r=-0.338, P<0.01$ respectively), and positively associated with the A/NA-Ghr ratio $(r=0.347, P<0.01$, and $r=0.334, P<0.05$ respectively) after adjustment for age, gender, and Tanner stage. Moreover, in these children, A-Ghr was also significantly associated with insulin and HOMAIR $(r=0.239, P<0.05$, and $r=0.219, P<0.05$ respectively) after adjustment for age, gender, and Tanner stage.

\section{Ghrelin profiles and MS}

Out of the 210 children included in the study, 39 (18.5\%) were identified as having MS. Compared with the children without MS, those with MS had a higher mean age (10.6 (95\% CI, 9.8-11.4) vs 9.1 (8.6-9.6) years; $P<0.05)$, as well as a BMI-SDS $(2.2(2.1-2.3)$ vs 0.95 (0.75-1.15); $P<0.0001)$, WC (78.5 (76.1-80.3) vs $70.0(68.7-71.8) \mathrm{cm} ; P<0.0001)$, systolic $\mathrm{BP}(115$ $(112-117)$ vs $110(108-111) \mathrm{mmHg} ; \mathrm{P}<0.0001)$,

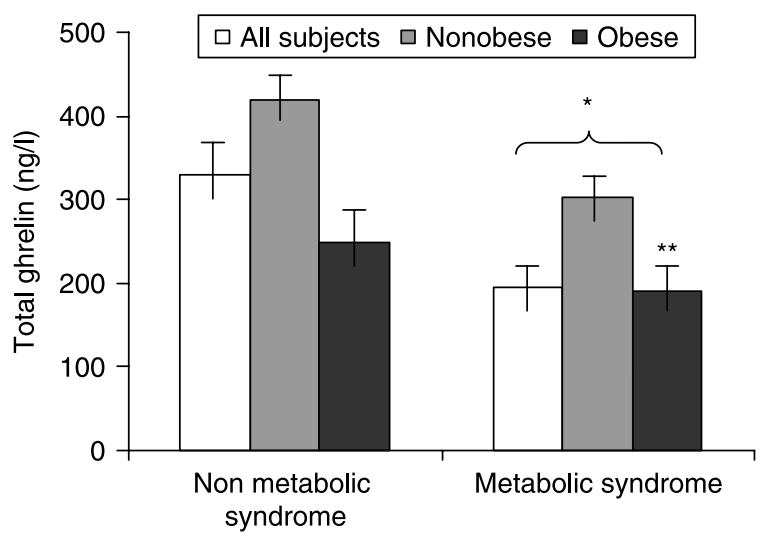

Figure 1 Geometric mean concentrations $(95 \% \mathrm{Cl})$ of total ghrelin in the entire population (white bars), normal weight children (gray bars), and obese patients (black bars) according to the presence of metabolic syndrome. ${ }^{*} P<0.0001$ versus nonmetabolic counterpart; ${ }^{*} P<0.05$ versus normal weight children with metabolic syndrome.

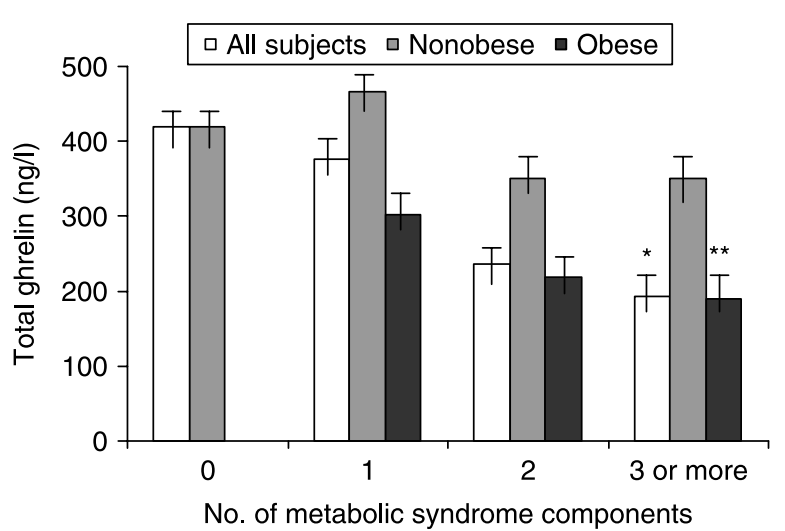

Figure 2 Geometric mean concentrations $(95 \% \mathrm{Cl}$ ) of total ghrelin in the entire population (white bars), normal weight children (gray bars), and obese patients (black bars) according to the number of metabolic syndrome components. ${ }^{*} P<0.0001$ and ${ }^{* *} P<0.001$ for trend.

diastolic BP (75 (72-77) vs 69 (68-70) $\mathrm{mmHg}$; $P<0.0001)$, triglycerides (183 (128-238) vs 82 $(74-90) \mathrm{mg} / \mathrm{dl} ; P<0.0001)$, insulin $(29(23-35)$ vs $12(11-13) \mathrm{mU} / \mathrm{l} ; P<0.0001)$, and HOMA-IR values (6.1 (4.9-7.4) vs $2.4(2.2-2.7) ; P<0.0001)$, but had lower HDL cholesterol values $(39(36-43)$ vs 47 $(45-49) \mathrm{mg} / \mathrm{dl} ; P<0.0001)$. Glucose and total cholesterol levels did not differ between the two groups. The mean concentrations of T-Ghr were significantly lower in patients with MS than in those without MS (194 (95\% CI, 160-235) vs 330 (296-365) ng/l; $P<0.0001 ;$ Fig. 1), and they decreased with the number of the components of MS (420 (347-508) vs $376(317-445)$ vs 236 (201-279) vs 193 (160-235) $\mathrm{ng} / \mathrm{l}$ ) in subjects with $0,1,2$, and $\geq 3$ components of MS, $P<0.0001$ for trend (Fig. 2). Similarly, NA-Ghr concentrations were significantly lower in patients with MS than in those without MS (83 (57-120) vs 196 (166-235) ng/l; $P<0.0001)$, and they decreased with the number of components of MS (311 (245-395) vs $230(172-311)$ vs $114(84-148)$ vs $83(55-119)$ ng/l) in subjects with $0,1,2$, and $\geq 3$ components of MS, $P<0.0001$ for trend. No significant differences were found for A-Ghr within the entire population (Figs 3 and 4). In contrast, the A/NA-Ghr ratio was significantly higher in all the patients with MS than in those without MS (100 (65-155) vs $44(36-53) \%$; $P<0.001)$; Fig. 5), and they increased with the number of the components of MS (26 (21-33) vs 41 (30-56) vs $73(51-104)$ vs $100(65-157) \%)$ in subjects with $0,1,2$, and $\geq 3$ components of MS, $P<0.0001$ for trend (Fig. 6).

Out of the 39 patients with MS, 30 (76.9\%) belonged to the group of obese children, and $9(23.0 \%)$ to the normal weight group. Compared with normal weight subjects with MS, obese children with MS had a similar mean age as well as mean values for systolic BP and diastolic BP and HDL cholesterol, but had higher values 


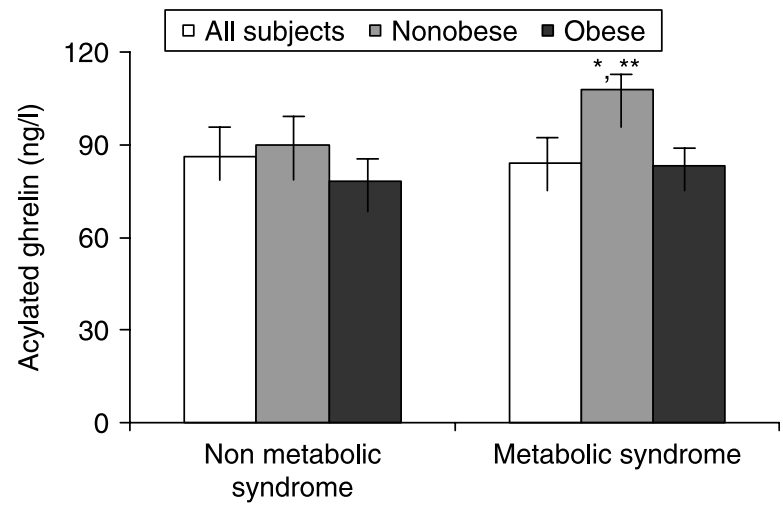

Figure 3 Geometric mean concentrations $(95 \% \mathrm{Cl})$ of acylated ghrelin in the entire population (white bars), normal weight children (gray bars), and obese patients (black bars) according to the presence of metabolic syndrome. ${ }^{\star} P<0.01$ versus nonmetabolic counterpart; ${ }^{* \star} P<0.01$ versus obese children with metabolic syndrome.

for triglycerides (189 (125-236) vs 130 (55-210) $\mathrm{mg} / \mathrm{dl} ; P<0.05)$, glucose $(4.85(4.69-5.01)$ vs 4.28 (3.97-4.58) $\mathrm{mmol} / \mathrm{l} ; \quad P<0.01$ ), and HOMA-IR $(5.7$ $(4.6-7.0)$ vs $4.4(3.0-6.9) ; P<0.05)$ and had lower values for total cholesterol (171 (154-188) vs 204 (146-262) mg/dl; $P<0.05$ ). T-Ghr (Fig. 1) and NA-Ghr were lower in obese children with MS than in normal weight patients with MS, while the A/NA-Ghr ratio was higher (Fig. 5). In contrast, A-Ghr was higher in normal weight children with MS than in obese patients with MS (Fig. 3).

We performed a multiple linear regression analysis to investigate the independent association of the single components of MS with ghrelin forms. WC, systolic BP, triglycerides, HDL cholesterol, glucose, and insulin values, along with age, gender, and Tanner stage, were included in the model. To avoid colinearity, diastolic BP and BMI were not included. WC (understandardized coefficient $(95 \% \mathrm{CI}),-0.104(-0.167$ to

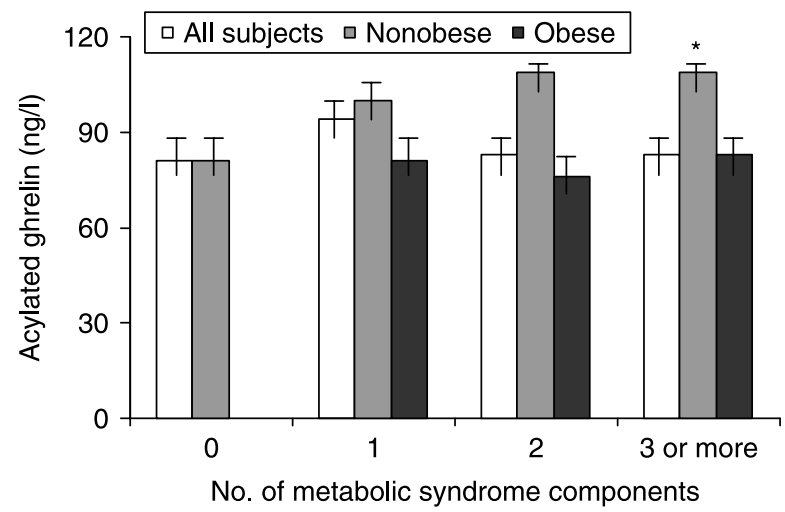

Figure 4 Geometric mean concentrations $(95 \% \mathrm{Cl})$ of acylated ghrelin in the entire population (white bars), normal weight children (gray bars), and obese patients (black bars) according to the number of metabolic syndrome components. ${ }^{\star} P<0.01$ for trend.

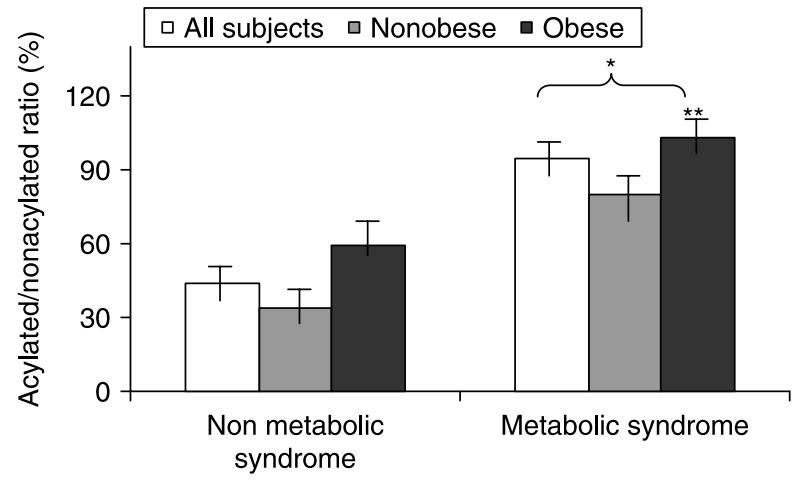

Figure 5 Geometric mean concentrations $(95 \% \mathrm{Cl})$ of acylated/ nonacylated ghrelin ratio in the entire population (white bars), normal weight children (gray bars) and obese patients (black bars) according to the presence of metabolic syndrome. ${ }^{\star} P<0.001$ versus nonmetabolic counterpart; ${ }^{\star \star} P<0.05$ versus normal weight children with metabolic syndrome.

$-0.042) ; P<0.05)$, systolic BP $(-0.013(-0.026$ to $-0.0001) ; P<0.05)$, triglycerides $(-0.002(-0.003$ to -0.001$) ; P<0.01)$, and insulin $(-0.126(-0.267$ to -0.011$) ; P<0.05)$ were independently and negatively associated with total ghrelin concentrations. In contrast, the A/NA-Ghr ratio was positively associated with systolic BP (0.032 (0.008-0.057); P<0.01), triglycerides $(0.002(0.0001-0.004) ; P<0.05)$, and insulin $(0.473(0.227-0.718) ; P<0.0001)$. If insulin was removed from the model, WC was significantly associated with the A/NA-Ghr ratio (0.04 (0.0020.077); $P<0.05)$.

\section{Multivariate regression analysis of the association between HOMA-IR values and plasma ghrelin forms}

Multiple linear regression analysis was used to evaluate the roles of ghrelin profiles as independent predictors of IR. As shown in Table 3, A-Ghr and the A/NA-Ghr ratios were significantly positively associated with HOMA-IR after adjustment for age, gender, Tanner stage, and BMI (or WC) as well as after adjustment for age, gender, Tanner stage, BMI (or WC), and other components of MS including systolic BP, triglycerides, and HDL cholesterol. In contrast, T-Ghr and NA-Ghr were negatively associated with HOMA-IR after correction for anthropometric and metabolic variables (Table 3 ).

\section{Discussion}

Recent research has focused on the role of ghrelin in the regulation of both insulin action and glucose homeostasis. Most available data indicate a negative association between total ghrelin and insulin concentrations $(5,39,40)$. However, with respect to IR, the relevance of NA versus A-Ghr has been assessed in very few studies 


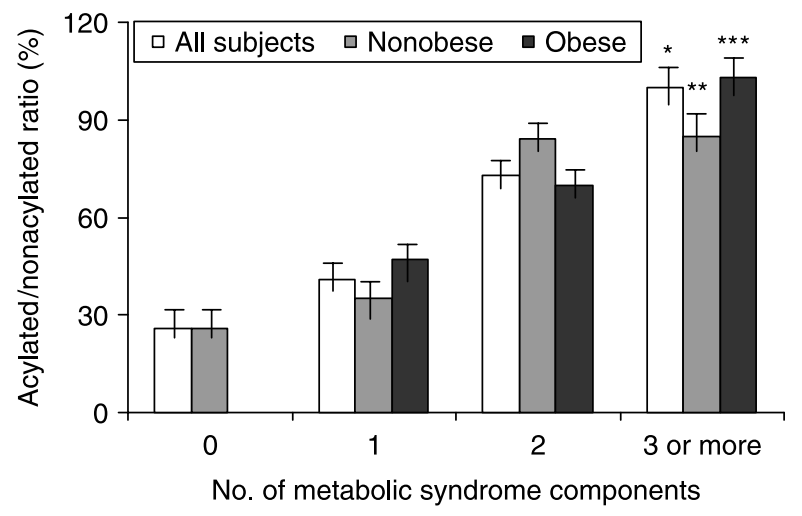

Figure 6 Geometric mean concentrations (95\% Cl) of acylated/ nonacylated ghrelin ratio in the entire population (white bars), normal weight children (gray bars), and obese patients (black bars) according to the number of metabolic syndrome components. ${ }^{\star} P<0.0001,{ }^{\star \star} P<0.001,{ }^{\star \star \star} P<0.01$ for trend.

performed in adult patients $(17,18,41)$. In a group of 60 overweight or obese postmenopausal women (31 insulin sensitive and 29 insulin resistant), St-Pierre et al. (18) demonstrated that both increased acylated ghrelin physiological concentrations and elevated A/NA-Ghr ratios were associated with IR. In a group of 45 adult MS patients, of whom 33 were obese and 12 were nonobese, Barazzoni et al. (17) demonstrated that plasma insulin and HOMA-IR were associated negatively with T-Ghr and NA-Ghr, but positively with A-Ghr and the A/NA-Ghr ratio. Compared to nonobese patients, obese MS patients had lower T-Ghr and NA-Ghr but comparable A-Ghr, and had a higher A/NA-Ghr ratio. BMI and WC were positively related to HOMA-IR. However, opposite associations between the A/NA-Ghr ratio and HOMA-IR remained significant after adjustment for sex and BMI (or WC). Yet, in the same study, Barazzoni et al. showed that ten obese individuals without MS (age-, sex-, BMI-, and WC-matched to obese MS patients) had lower T-Ghr but higher A-Ghr than 15 age- and sex-matched healthy nonobese controls. T-Ghr and A-Ghr were comparable in obese individuals with or without MS. In a very recent study (41) involving 80 adult subjects
(19 obese with type 2 diabetes; 20 obese with impaired glucose tolerance; 20 obese with normoglycemia; and 21 lean with normoglycemia), Rodriguez et al. demonstrated that obese subjects had increased circulating concentrations of A-Ghr and decreased circulating concentrations of desacyl ghrelin than lean individuals. Furthermore, acylated ghrelin values were higher in obese individuals with impaired glucose tolerance and type 2 diabetes than in obese normoglycemic patients. No effect of glucose tolerance or diabetes was observed in circulating desacyl ghrelin concentrations. A highly significant positive correlation was observed between acylated ghrelin and BMI, WC, and HOMA, whereas desacyl ghrelin showed a strong negative correlation with these parameters.

To our knowledge, we are the first to investigate the relevance of ghrelin profiles, including A-Ghr and NA-Ghr, and the ratio of A/NA-Ghr in the context of IR in a large population of obese children as well as of normal weight children. The results of the present study show that both T-Ghr and NA-Ghr are inversely related with IR, while A-Ghr and the ratio of A/NA-Ghr are positively associated with IR, independently of anthropometric and metabolic variables. We have also demonstrated that obese children with MS have marked alterations in plasma ghrelin profile than obese children without MS; in particular, T-Ghr and NA-Ghr were lower, while the fraction of acylated circulating hormone relative to nonacylated circulating hormone was higher. Moreover, T-Ghr and NA-Ghr decreased, while A/NA-Ghr ratio increased monotonically with the number of MS components.

Interestingly, there was also an alteration of ghrelin secretion profiles in normal weight children with MS. In fact, T-Ghr and NA-Ghr were lower, while A-Ghr and the A/NA-Ghr ratio were higher in normal weight subjects with MS than in normal weight counterparts without MS. A positive correlation between A-Ghr and the A/NA-Ghr ratio and insulin levels as well as IR index was observed in these subjects. In agreement with previous findings in adults (17), NWMA children had a larger WC than healthy normal weight subjects despite similar BMI and total fat mass. Recent evidence in children shows that WC is more closely associated with

Table 3 Multivariate linear regression analyses of plasma ghrelin forms (as independent variables) and homeostasis model assessment of insulin resistance (HOMA-IR) (dependent variable) in the entire study population.

Unstandardized coefficient, $\boldsymbol{b}^{\text {a }}(95 \% \mathrm{Cl})$

Unstandardized coefficient, $\boldsymbol{b}^{\mathrm{b}}(95 \% \mathrm{Cl})$

\begin{tabular}{lcc}
\hline Total ghrelin $(\mathrm{ng} / \mathrm{l})$ & $-0.141(-0.275 \text { to }-0.008)^{\star}$ & $-0.133(-0.269 \text { to }-0.003)^{\star}$ \\
Nonacylated ghrelin $(\mathrm{ng} / \mathrm{l})$ & $-0.100(-0.171 \text { to }-0.028)^{\ddagger}$ & $-0.092(-0.166 \text { to }-0.018)^{\star}$ \\
Acylated ghrelin $(\mathrm{ng} / \mathrm{l})$ & $0.168(0.016-0.321)^{\star}$ & $0.165(0.012-0.317)^{\star}$ \\
Acylated to nonacylated ghrelin ratio (\%) & $0.104(0.042-0.166)^{\dagger}$ & $0.098(0.035-0.161)^{\ddagger}$
\end{tabular}

Total ghrelin, acylated ghrelin, nonacylated ghrelin, acylated to nonacylated ghrelin ratio, and HOMA-IR were log transformed for the best fit. ${ }^{\star} P<0.05 ;{ }^{\dagger} P<0.01 ;{ }^{\ddagger} P<0.001$.

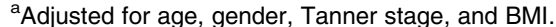

${ }^{\mathrm{b}}$ Adjusted for age, gender, Tanner stage, and BMI and other components of metabolic syndrome including triglycerides, HDL cholesterol, and systolic BP. Similar results were observed when adjusting for waist circumference (instead of BMI). 
visceral fat, whereas BMI is more associated with subcutaneous fat (42). Similarly, visceral fat (as measured by magnetic resonance imaging), but not BMI or waist-hip ratio, has been associated with fasting insulin and triglycerides in obese adolescent girls (43). Given that the visceral adipose tissue constitutes a source of ghrelin (44), it has been suggested that abdominal fat accumulation may contribute to the relative or absolute A-Ghr excess, which may in part account for the interaction between visceral fat and IR in MS patients $(17,41)$. However, despite adjustment for WC (as a surrogate and indirect index of visceral obesity), ghrelin forms remained independently associated with IR.

Our results of a differential association of A-Ghr and NA-Ghr with IR are supported by several in vitro and in vivo studies. Ghrelin forms had an opposite impact on glucose output in primary cultures of porcine hepatocytes (13). In addition, NA-Ghr was shown to counteract the influence of A-Ghr on insulin secretion and on glucose metabolism in young male volunteers (14). Furthermore, A-Ghr and NA-Ghr displayed correlations of opposite signs with variables regarding cholesterol metabolism and hepatic function, confirming the divergent effects of both forms on metabolic processes (41). The relation of A-Ghr to NA-Ghr may have a clinical significance in metabolic disorders. The current results suggest that the A/NA-Ghr balance may represent a fine-tuning mechanism that can modulate insulin action. Unbalanced plasma concentrations of ghrelin forms and a relative A-Ghr excess may negatively modulate insulin action in obese and normal weight children, thus contributing to the association of IR with MS. Factors regulating circulating A-Ghr levels are largely unknown. Putative mechanisms for elevated A-Ghr include increased ghrelin acylation and/or increased ghrelin synthesis and secretion. Increased availability of fatty acids may enhance hormone acylation in mice (45). Recently, Yang et al. (46) have identified a novel enzyme implicated in the $n$-octanoylation of ghrelin, namely ghrelin $O$-acyltransferase, which is expressed in the major ghrelin-secreting tissues, such as the stomach and the intestine. Recent evidence in rodents also suggests a direct role of dietary lipids in ghrelin acylation (47). Therefore, it seems plausible that obesity, in particular visceral adiposity, may influence the expression and/or activity of this acyltransferase, leading to elevated plasma A-Ghr concentrations even in the presence of normal total body fat mass.

In adults, low total ghrelin concentration has been associated with the single features of MS, including elevated BP, hypertriglyceridemia, high fasting blood glucose, and low HDL cholesterol (48-51). Yet, the relationship between ghrelin levels and metabolic abnormalities as a cluster per se has also been reported (51). Because adiposity influences all other features of the MS, it has been suggested that low ghrelin in MS could reflect only the obesity state. Langenberg et al.
(49) demonstrated that systolic BP and diastolic BP, fasting and postchallenge insulin, HOMA-IR, HDL cholesterol, and triglycerides were associated with ghrelin before and after adjustment for age and sex. Adjustment for BMI attenuated most associations; only fasting and postchallenge insulin and HDL cholesterol remained significantly associated with ghrelin. Furthermore, Ukkola et al. (51) reported that the association of HDL cholesterol and fasting blood glucose with plasma ghrelin levels remained significant after adjustment for BMI. Yet, ghrelin levels decreased with an increase in the number of metabolic abnormalities. Finally, in the study by Ukkola et al. low ghrelin was a significant predictor of MS as a cluster per se after adjustment for age and sex (51). Our results confirm and expand on the above-mentioned findings. We have found that single components of MS were independently and inversely associated with total ghrelin levels. We have also demonstrated that single components of MS were independently and positively associated with the A/NA-Ghr ratio.

In conclusion, our data indicate that relative A-Ghr excess is associated with IR independently of obesity as well as of other components of MS. Further longitudinal investigations will be needed to elucidate whether dysregulation of ghrelin secretion profiles in obese children as well as in normal weight children with metabolic abnormalities may influence the long-term metabolic and cardiovascular outcomes.

\section{Declaration of interest}

There is no conflict of interest that could be perceived as prejudicing the impartiality of the research reported.

\section{Funding}

This research did not receive any specific grant from any funding agency in the public, commercial, or not-for-profit sector.

\section{Author contribution statement}

The authors' responsibilities were as follows: L Pacifico participated in the design of the study, data collection and analysis, and writing of the manuscript; E Poggiogalle and F Costantino participated in the collection and analysis of data; F Ferraro assisted with the data analysis and in drafting the manuscript; C Anania and F Chiarelli provided significant advice on all the aspects of the study; and C Chiesa participated in the design of the study, data analysis, and writing of the manuscript. All the authors participated in the critical review and in the final approval of the manuscript.

\section{References}

1 Van der Lely AJ, Tschop M, Heiman ML \& Ghigo E. Biological, physiological, pathophysiological, and pharmacological aspects of ghrelin. Endocrine Reviews 200425 426-457.

2 Kojima M, Hosoda H, Date Y, Nakazato M, Matsuo H \& Kangawa K. Ghrelin is a growth-hormone-releasing acylated peptide from stomach. Nature $1999 \mathbf{4 0 2} 656-660$. 
3 Wiedmer P, Nogueiras R, Broglio F, D’Alessio D \& Tschop MH. Ghrelin, obesity and diabetes. Nature Clinical Practice. Endocrinology and Metabolism 20073 705-712.

4 Tschöp M, Weyer C, Tataranni PA, Devanarayan V, Ravussin E \& Heiman ML. Circulating ghrelin levels are decreased in human obesity. Diabetes 200150 707-709.

5 Ikezaki A, Hosoda H, Ito K, Iwama S, Miura N, Matsuoka H, Kondo C, Kojima M, Kangawa K \& Sugihara S. Fasting plasma ghrelin levels are negatively correlated with insulin resistance and PAI-1, but not with leptin, in obese children and adolescents. Diabetes 200251 3408-3411.

6 Shiiya T, Nakazato M, Mizuta M, Date Y, Mondal MS, Tanaka M, Nozoe S, Hosoda H, Kangawa K \& Matsukura S. Plasma ghrelin levels in lean and obese humans and the effect of glucose on ghrelin secretion. Journal of Clinical Endocrinology and Metabolism 200287 240-244.

7 Broglio F, Arvat E, Benso A, Gottero C, Muccioli G, Papotti M, van der Lely AJ, Deghenghi R \& Ghigo E. Ghrelin, a natura GH secretagogue produced by the stomach, induces hyperglycemia and reduces insulin secretion in humans. Journal of Clinical Endocrinology and Metabolism 200186 5083-5086.

8 Tannous dit El Khoury D, Obeid O, Azar ST \& Hwalla N. Variations in postprandial ghrelin status following ingestion of highcarbohydrate, high-fat, and high-protein meals in males. Annals of Nutrition and Metabolism $2006 \mathbf{5 0}$ 260-269.

9 Quader SS, Lundquist I, Ekelund M, Håkanson R \& Salehi A. Ghrelin activates neuronal constitutive nitric oxide synthase in pancreatic islet cells while inhibiting insulin release and stimulating glucagon release. Regulatory Peptides $2005 \mathbf{1 2 8}$ 51-56.

10 Westergaard ET, Hansen TK, Gormsen LC, Jakobsen P, Moller N, Cristiansen JS \& Jorgensen JO. Constant intravenous ghrelin infusion in healthy young men: clinical pharmacokinetics and metabolic effects. American Journal of Physiology. Endocrinology and Metabolism 2007292 E1829-E1836.

11 Guido M, Romualdi D, De Marinis L, Porcelli T, Giuliani M, Costantini B \& Lanzone A. Administration of exogenous ghrelin in obese patients with polycystic ovary syndrome: effects on plasma levels of growth hormone, glucose, and insulin. Fertility and Sterility $2007 \mathbf{8 8} 125-130$.

12 Foster-Schubert KE, Overduin J, Prudom CE, Liu J, Callahan HS, Gaylinn BD, Thorner MO \& Cummings DE. Acyl and total ghrelin are suppressed strongly by ingested proteins, weakly by lipids, and biphasically by carbohydrates. Journal of Clinical Endocrinology and Metabolism 200893 1971-1979.

13 Gauna C, Delhanty PJ, Hofland LJ, Janssen JA, Broglio F, Ross RJ, Ghigo E \& van der Lely AJ. Ghrelin stimulates, whereas desoctanoyl ghrelin inhibits glucose output by primary hepatocytes. Journal of Clinical Endocrinology and Metabolism 200590 1055-1060.

14 Broglio F, Gottero C, Prodam F, Gauna C, Muccioli G, Papotti M, Abribat T, Van Der Lely AJ \& Ghigo E. Non-acylated ghrelin counteracts the metabolic but not the neuroendocrine response to acylated ghrelin in humans. Journal of Clinical Endocrinology and Metabolism 200489 3062-3065.

15 Gauna C, Meyler FM, Janssen JA, Delhanty PJ, Abribat T, van Koetsveld P, Hofland LJ, Broglio F, Ghigo E \& van der Lely AJ. Administration of acylated ghrelin reduces insulin sensitivity, whereas the combination of acylated plus unacylated ghrelin strongly improves insulin sensitivity. Journal of Clinical Endocrinology and Metabolism 200489 5035-5042.

16 Gauna C, Kiewiet RM, Janssen JA, van de Zande B, Delhanty PJ, Ghigo E, Hofland LJ, Themmen AP \& van der Lely AJ. Unacylated ghrelin acts as a potent insulin secretagogue in glucose-stimulated conditions. American Journal of Physiology. Endocrinology and Metabolism 2007293 E697-E704.

17 Barazzoni R, Zanetti M, Ferreira C, Vinci P, Pirulli A, Mucci M, Dore F, Fonda M, Ciocchi B, Cattin L \& Guarnieri G. Relationships between desacylated and acylated ghrelin and insulin sensitivity in the metabolic syndrome. Journal of Clinical Endocrinology and Metabolism 200792 3935-3940.
18 St-Pierre DH, Karelis AD, Coderre L, Malita F, Fontaine J, Mignault D, Brochu M, Bastard JP, Cianflone K, Doucet E, Imbeault P \& Rabasa-Lhoret R. Association of acylated and nonacylated ghrelin with insulin sensitivity in overweight and obese postmenopausal women. Journal of Clinical Endocrinology and Metabolism 200792 264-269.

19 Mackelvie KJ, Meneilly GS, Elahi D, Wong ACK, Barr SI \& Chanoine J-P. Regulation of appetite in lean and obese adolescents after exercise: role of acylated and desacyl ghrelin. Journal of Clinical Endocrinology and Metabolism 200792 648-654.

20 Hauffa BP, Haase K, Range IM, Unger N, Mann K \& Petersenn S. The effect of growth hormone on the response of total and acylated ghrelin to a standardized oral glucose load, and insulin resistance in children with Prader-Willi syndrome. Journal of Clinical Endocrinology and Metabolism 200792 834-840.

21 Steinberger J \& Daniels SR. Obesity, insulin resistance, diabetes and cardiovascular risk in children: an American Heart Association scientific statement from the Atherosclerosis, Hypertension, and Obesity in the Young Committee (Council on Cardiovascular Disease of the Young) and the Diabetes Committee (Council on Nutrition, Physical Activity, and Metabolism). Circulation 2003 107 1448-1453.

22 Baker JL, Olsen LW \& Sorensen TI. Childhood body-mass index and the risk of coronary heart disease in adulthood. New England Journal of Medicine 200723 2329-2337.

23 Weiss R, Dziura J, Burgert TS, Tamborlane WV, Taksali SE, Yeckel CW, Allen K, Lopes M, Savoye M, Morrison J, Sherwin RS \& Caprio S. Obesity and the metabolic syndrome in children and adolescents. New England Journal of Medicine $20043 \mathbf{3 0 0}$ 2362-2374.

24 Chiarelli F \& Marcovecchio ML. Insulin resistance and obesity in childhood. European Journal of Endocrinology 2008159 S67-S74.

25 Cacciari E, Milani S, Balsamo A, Dammacco F, De Luca F, Chiarelli F, Pasquino AM, Tonini G \& Vanelli M. Italian crosssectional growth charts for height, weight and BMI $(6-20 \mathrm{y})$. European Journal of Clinical Nutrition 200256 171-180.

26 Marshall WA \& Tanner JM. Variations in pattern of pubertal changes in girls. Archives of Disease in Childhood $1969 \mathbf{4 4}$ 291-303.

27 Marshall WA \& Tanner JM. Variations in pattern of pubertal changes in boys. Archives of Disease in Childhood 197045 13-23.

28 Cole TJ, Bellizzi MC, Flegal KM \& Dietz WH. Establishing a standard definition for child overweight and obesity worldwide: international survey. BMJ 2000320 1240-1243.

29 Invitti C, Maffeis C, Gilardini L, Pontiggia B, Mazzilli G, Girola A, Sartorio A, Morabito F \& Viberti GC. Metabolic syndrome in obese Caucasian children: prevalence using WHO-derived criteria and association with nontraditional cardiovascular risk factors. International Journal of Obesity $200630627-633$.

30 Steinberger J, Daniels SR, Eckel RH, Hayman L, Lustig RH, McCrindle B \& Mietus-Snyder ML, American Heart Association Atherosclerosis, Hypertension, and Obesity in the Young Committee of the Council on Cardiovascular Disease in the Young; Council on Cardiovascular Nursing; and Council on Nutrition, Physical Activity, and Metabolism. Progress and challenges in metabolic syndrome in children and adolescents. Circulation $2009119628-647$.

31 Zimmet P, Alberti KG, Kaufman F, Tajima N, Silink M, Arslanian S, Wong G, Bennett P, Shaw J \& Caprio S, IDF Consensus Group. The metabolic syndrome in children and adolescents - an IDF Consensus Report. Pediatric Diabetes 20078 299-306.

32 Third Report of the National Cholesterol Education Program (NCEP). Expert panel on detection, evaluation, and treatment of high blood cholesterol in adults (Adult Treatment Panel III) final report. Circulation $2002 \mathbf{1 0 6} 3143-3421$.

33 Alberti KG \& Zimmet PZ. Definition, diagnosis and classification of diabetes mellitus and its complications. 1. Diagnosis and classification of diabetes mellitus provisional report of a WHO consultation. Diabetic Medicine 199815 539-553. 
34 Goodman E, Daniels SR, Meigs JB \& Dolan LM. Instability in the diagnosis of metabolic syndrome in adolescents. Circulation 2007 $1152316-2322$.

35 Zannolli R \& Morgese G. Waist percentiles: a simple test for atherogenic disease? Acta Paediatrica 199685 1368-1369.

36 American Academy of Pediatrics. National Cholesterol Education Program: report of the expert panel on blood cholesterol levels in children and adolescents. Pediatrics 199289 525-584.

37 National High Blood Pressure Education Program Working Group on High Blood Pressure in Children and Adolescents. The fourth report on the diagnosis, evaluation, and treatment of high blood pressure in children and adolescents. Pediatrics 2004114 555-576.

38 Allard P, Delvin EE, Paradis G, Hanley JA, O’Loughlin J, Lavallée C, Levy E \& Lambert M. Distribution of fasting plasma insulin, free fatty acids, and glucose concentrations and of homeostasis model assessment of insulin resistance in a representative sample of Quebec children and adolescents. Clinical Chemistry $2003 \mathbf{4 9}$ 644-649.

39 Purnell JQ, Weigle DS, Breen P \& Cummings DE. Ghrelin levels correlate with insulin levels, insulin resistance, and high-density lipoprotein cholesterol, but not with gender, menopausal status, or cortisol levels in humans. Journal of Clinical Endocrinology and Metabolism $2003 \mathbf{8 8} 5747-5752$.

40 McLaughlin T, Abbasi F, Lamendola C, Frayo RS \& Cummings DE. Plasma ghrelin concentrations are decreased in insulin-resistant obese adults relative to equally obese insulin-sensitive controls. Journal of Clinical Endocrinology and Metabolism $2004 \mathbf{8 9}$ 1630-1635.

41 Rodriguez A, Gómez-Ambrosi J, Catalán V, Gil MJ, Becerril S, Sáinz N, Silva C, Salvador J, Colina I \& Frühbeck G. Acylated and desacyl ghrelin stimulate lipid accumulation in human visceral adipocytes. International Journal of Obesity 200953 541-552.

42 Brambilla P, Bedogni G, Moreno LA, Goran MI, Gutin B, Fox KR, Peters DM, Barbeau P, De Simone M \& Pietrobelli A. Crossvalidation of anthropometry against magnetic resonance imaging for the assessment of visceral and subcutaneous adipose tissue in children. International Journal of Obesity 200630 23-30.
43 Caprio S, Hyman LD, McCarthy S, Lange R, Bronson M \& Tamborlane WV. Fat distribution and cardiovascular risk factors in obese adolescent girls: importance of the intraabdominal fat depot. American Journal of Clinical Nutrition $19966412-17$.

44 Knerr J, Herzog D, Rauh M, Kasher W \& Horbach T. Leptin and ghrelin expression in adipose tissues and serum levels in gastric banding patients. European Journal of Clinical Investigation 200636 389-394.

45 Nishi Y, Hiejima H, Hosoda H, Kaiya H, Mori K, Fukue Y, Yanase T, Nawata H, Kangawa K \& Kojima M. Ingested medium-chain fatty acids are directly utilized for the acyl modification of ghrelin. Endocrinology $20051462255-2264$.

46 Yang J, Brown MS, Liang G, Grishin NV \& Goldstein JL. Identification of the acyl-transferase that octanoylates ghrelin, an appetite-stimulating peptide hormone. Cell $2008 \mathbf{1 3 2}$ 387-396.

47 Kirchner H, Gutierrez JA, Solenberg PJ, Pfluger PT, Czyzyk TA, Willency JA, Schürmann A, Joost H-G, Jandacek RJ, Hale JE, Heiman ML \& Tschöp MT. Goat links dietary lipids with the endocrine control of energy balance. Nature Medicine 200915 $741-745$.

48 Ukkola O. Ghrelin and metabolic disorders. Current Protein and Peptide Science 200910 2-7.

49 Langenberg C, Bergstrom J, Laughlin GA \& Barrett-Connor E. Ghrelin and the metabolic syndrome in older adults. Journal of Clinical Endocrinology and Metabolism 200590 6448-6453.

50 Pöykkö SM, Kellokoski E, Hörkkö S, Kauma H, Kesäniemi YA \& Ukkola O. Low plasma ghrelin is associated with insulin resistance, hypertension, and the prevalence of type 2 diabetes. Diabetes 2003 52 2546-2553.

51 Ukkola O, Pöykkö SM \& Kesäniemi YA. Low plasma ghrelin concentration is an indicator of the metabolic syndrome. Annals of Medicine $20063 \mathbf{8} 274-279$.

Received 10 September 2009

Accepted 16 September 2009 